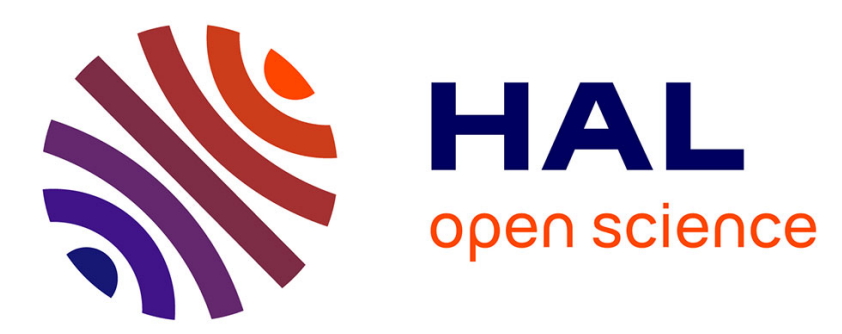

\title{
Despiking SEEG signals reveals dynamics of gamma band preictal activity
}

Nawel Jmail, Martine Gavaret, F Bartolomei, Christian-G Bénar

\section{To cite this version:}

Nawel Jmail, Martine Gavaret, F Bartolomei, Christian-G Bénar. Despiking SEEG signals reveals dynamics of gamma band preictal activity. Physiological Measurement, 2017, 38 (2), pp.N42-N56. 10.1088/1361-6579/38/2/N42 . hal-03576849

\section{HAL Id: hal-03576849 \\ https://hal.science/hal-03576849}

Submitted on 16 Feb 2022

HAL is a multi-disciplinary open access archive for the deposit and dissemination of scientific research documents, whether they are published or not. The documents may come from teaching and research institutions in France or abroad, or from public or private research centers.
L'archive ouverte pluridisciplinaire HAL, est destinée au dépôt et à la diffusion de documents scientifiques de niveau recherche, publiés ou non, émanant des établissements d'enseignement et de recherche français ou étrangers, des laboratoires publics ou privés.

\section{(ㅇ)(1) $\$$}

Distributed under a Creative Commons Attribution - NonCommercial - NoDerivatives| 4.0 


\title{
Despiking SEEG signals reveals dynamics of gamma band preictal activity
}

\author{
Nawel Jmail a , Martine Gavaret ${ }^{\mathrm{b}, \mathrm{c}}, \mathrm{F}$ Bartolomei ${ }^{\mathrm{b}, \mathrm{c}}$,Christian-G. Bénar ${ }^{\mathrm{b}}$
}

a. Sfax University, ENIS, MIRACL, Tunisia

b. Aix Marseille Univ, Inserm, INS, Institut de Neurosciences des Systèmes, Marseille, France

c. APHM, Hôpital de la Timone, Neurophysiologie clinique, Marseille, France

\section{Corresponding author:}

Christian George Bénar, INSERM UMR 1106, Institut de Neurosciences des Systèmes, Marseille, France-13005

Tel: +334913855 62. Fax: +33491789914

E-mail address: christian.benar@univ-amu.fr

\begin{abstract}
:
Interictal epileptiform discharges, or "interictal spikes", are the hallmark of epilepsy. Still, there is growing evidence that oscillatory activity - whether in the gamma band $(30-120 \mathrm{~Hz})$ or at higher frequencies is another important marker of hyperexcitable tissues. A major difficulty arises from the fact that interictal spikes and oscillations overlap in the frequency domain. This hampers the correct delineation of the cortex producing pathological oscillations by simple filtering. Here, we propose a nonlinear technique for fitting the spike waveform in order to remove it, resulting in a "despiked" signal. This strategy was first applied to simulated data inspired from real stereo-electroencephalographic (SEEG) signals, then to real data. We show that despiking leads to a better space-time-frequency analysis of the oscillatory part of the signal. Thus, in the real SEEG signals, the spatio-temporal maps show a buildup of gamma oscillations during the preictal period in the despiked signals, whereas in the original signals this activity is masked by spikes. Despiking is thus a promising venue for a better characterization of oscillatory activity in electrophysiology of epilepsy.
\end{abstract}




\section{Introduction}

Electrophysiological brain signals in epilepsy show a complex mixture of patterns at different frequencies, reflecting the pathological activation of multiple neuronal networks. Signals can be broadly divided into two states, the ictal state (corresponding to seizures) and the interictal state (between seizures). The question arises of the existence of a third state - a "preictal" phase - where activity could in some cases build up and lead to the seizure (Wendling et al. 2005; Schwartz et al. 2011). In the interictal state, the most classical marker of epilepsy is the interictal epileptiform discharge (IED), or "interictal spike", a sharp transient lasting less than 50 ms (Gotman and Gloor 1976). Another important pattern, particularly visible in focal cortical dysplasia, is that of gamma-band epileptic oscillations, in approximately the $35-120 \mathrm{~Hz}$ band $^{1}$ (Hirai et al. 1999). Recently, much interest has been directed towards high frequency oscillations, the "fast ripples", in the $250-500 \mathrm{~Hz}$ band, which have been shown to be a good marker of the epileptogenic zone (Urrestarazu et al. 2007; Jacobs et al. 2008; Crépon et al. 2010). In the ictal period, a pattern of high interest is the fast discharge, which corresponds to a flattening of the signal, i.e., an increase in the proportion of high frequencies in the signals (Bartolomei et al. 2008). This pattern is classically considered as defining the seizure onset zone. Recently, automatic indices have been proposed in order to define the epileptogenic zone, based on the fast discharge detection or the contrast between high and low frequencies (Wendling et al. 2003; Bartolomei et al. 2008; David et al. 2011). Therefore, the question arises of the relationships between the high frequencies activities as measured in the interictal or pre-ictal state and those observed at the seizure onset.

A major difficulty in this task arises from the fact that interictal/preictal spikes and high frequency oscillations overlap in the frequency domain. Indeed, the extraction of the "pure" oscillatory component, i.e. not contaminated from filter ringing arising from spikes is a difficult issue. In particular, band-pass filtering spikes - which are sharp events by definitionproduce spurious oscillatory patterns corresponding to the impulse response of the filter (Bénar et al. 2010). In the time frequency domain, the oscillatory and spike parts can be

\footnotetext{
${ }^{1}$ Limits of the gamma band are not clearly defined. Typically, the low gamma ranges from around $35 \mathrm{~Hz}$ to around $80 \mathrm{~Hz}$, the high gamma band from around $80 \mathrm{~Hz}$ to $120 \mathrm{~Hz}$.
} 
better discriminated, but there can still be overlaps in the time-frequency domain (Ayoubian et al. 2013). Dictionary-based filtering, such as matching pursuit or basis pursuit are in theory quite robust to the overlap, but require that the (theoretical) dictionary be well matched to the real signals (Jmail et al. 2011).

The objective of this study was to propose a signal processing strategy for removing the epileptic spikes from the intracerebral signals. We show that this leads to better characterization of the regions involved in actual high frequency oscillations, in particular in the preictal-to-ictal transition. We tested the method on simulated data for different level of jitter between spikes and oscillations, and on real stereo-electroencephalographic EEG (SEEG) recorded in one patient during presurgical evaluation of epilepsy. Firstly, we describe the simulated and real datasets, and the algorithm for separating spikes and oscillations. Secondly, we depict the results obtained for both simulated and real data in terms of reconstruction accuracy and network measures. Lastly, we present conclusions and future directions.

\section{Materials and methods}

All signal processing was performed with the Matlab software (Mathworks, Natick, MA), version 2015a.

\subsection{Simulations}

We constructed simulations that reproduce a preictal series of epileptiform discharges, inspired by real SEEG traces, with a sampling rate set to $512 \mathrm{~Hz}$. In the real data (see 2.2), we observed oscillations at different frequencies in the preictal period. Within the gamma band, oscillatory bursts were observed around $55 \mathrm{~Hz}$ and around $85 \mathrm{~Hz}$ (Supp. Fig. 1). Simulated data consisted of four components, corresponding to spikes, oscillations, seizure and noise. 100 realizations were constructed, one realization is shown on Figure 1.

Spikes were simulated as a sum of two gamma functions, using the $\operatorname{gampdf}(x, a, b)$ function in Matlab. With time variable $x$ in samples, we set $(a, b)$ to $(9.8,3.9)$ in channel $1,(7.8,2)$ in channel $2,(11.7,3.9)$ in channel 3 and $(11.7,2)$ in channel 4 . Amplitudes were set to 10 , with fluctuation ranging from $10 \%$ to $25 \%$ (uniform distribution), except for the first gamma 
function which presented no fluctuation. Time of occurrence of spikes was every $0.8 \mathrm{~s}$, with jitter ranging between 40 and $0 \mathrm{~ms}$, except for the first gamma function.

Oscillations were simulated as oscillatory pulses, using the gausspuls $(t, f c, b w)$ function of matlab, with $t$ time, $f c$ frequency of oscillation set to $55 \mathrm{~Hz}$ in channel 3 and $85 \mathrm{~Hz}$ in channel 4 and bw fractional bandwidth was set to 0.15 (corresponding to 7 periods of oscillations in the segment corresponding to the full-width half maximum). Amplitude fluctuations were set to $25 \%$ and $15 \%$ in channel 3 and 4 respectively, maximum temporal jitter to $50 \mathrm{~ms}$ and $40 \mathrm{~ms}$ in channel 3 and 4 respectively. The seizure part was simulated as two sine waves at $55 \mathrm{~Hz}$ in channel 3 and $85 \mathrm{~Hz}$ in channel 4, with envelope set to a Tukey window (tukeywin function of Matlab).

Noise was obtained by smoothing random Gaussian noise (function randn of Matlab) with a moving average of 10 points. Noise was set to an amplitude of 2 during the spike period, and 1 during the seizure period. A smooth transition between the two periods was ensured thanks to a half Tukey window, in order to avoid filter ringing arising from abrupt transitions.

\subsection{Real signals}

We selected presurgical intracerebral signals (stereo-electroencephalography, SEEG) in one patient with drug-resistant focal epilepsy from the Clinical Neurophysiology Department of the Timone hospital in Marseille. This epilepsy was symptomatic of a focal cortical dysplasia localized in the right occipito-temporal junction. The placement of depth electrodes was based upon available non-invasive information providing hypotheses about the localization of the epileptogenic zone. This patient presented a focal cortical dysplasia in the right lateral occipito-temporal junction. The SEEG data was acquired on a Deltamed System, sampled at $256 \mathrm{~Hz}$, with anti-aliasing low-pass analog filter set to $85 \mathrm{~Hz}$. This particular SEEG recording was selected for the current study because it presented clear preictal patterns with regular spiking and visible epileptic oscillations. SEEG was using eight multi-contact depth electrodes (diameter $0.8 \mathrm{~mm}, 10$ to 15 contacts, each $2 \mathrm{~mm}$ long with an inter-contact distance of 1.5 $\mathrm{mm})$, positioned in the right hemisphere, implanted according to Talairach's stereotaxic method (Talairach and Bancaud 1973). Patients signed informed consent, and the study was 
approved by the Institutional Review board (IRB00003888) of INSERM (IORG0003254, FWA00005831).

\subsection{Spike modeling}

We first detected spikes as events with high amplitude (in absolute value). In order to emphasize the transient part of the spikes, signals were first filtered in the $5 \mathrm{~Hz}-40 \mathrm{~Hz}$ band. Amplitude thresholds (on the signed values) were found based on data quartiles for each channel:

$$
\begin{aligned}
& t h r_{\text {high }}=Q_{0.5}+d\left(Q_{0.75}-Q_{0.25}\right) \\
& t h r_{\text {low }}=Q_{0.5}-d\left(Q_{0.75}-Q_{0.25}\right)
\end{aligned}
$$

With $Q p$ value in the distribution of amplitudes corresponding to the $p$ percentile, and $d$ distance parameter to be tuned heuristically. Typically, we found a value of 3 to be useful in most cases. Detections were found as the local peaks in the signal passing the amplitude thresholds, with a minimum distance between consecutive peaks set to $10 \mathrm{~ms}$ (function 'findpeaks' of Matlab). Then, for each detection, a model consisting of a modified Gaussian function was fitted non-linearly to the data:

$G(t, a, b, \gamma)=\left\{\begin{array}{r}-A e^{-\frac{[(t-a) * \gamma]^{2}}{b}}, t<a \\ A e^{-\frac{[(t-a) / \gamma]^{2}}{b}}, t \geq a\end{array}\right.$

With $A$ amplitude, $t$ time, $a$ shift parameter (corresponding to zero of time), $b$ scale parameters (width of the model function), $\gamma$ asymmetry parameter ( 1 corresponds to the original - symmetric - Gaussian function). In order to estimate the parameters $(a, b, v)$ corresponding to the best fit, we used the non-linear simplex method (fminsearch from Matlab). Parameter $A$ was fitted linearly by a least square method, with all other parameters fixed. The whole procedure results in a model of the spike components of the signal.

\subsection{Spatio-temporal analysis}

Time-frequency analysis was performed based on a Morlet wavelet transform with oscillation parameter $\xi=5$ (Bénar et al. 2009). Data were then presented under the form of 
spatio-temporal maps (\#channels $\mathrm{x}$ \#time points). In a first representation, data were filtered in the frequency band of the oscillations (David et al. 2011), using the EEGLAB matlab function "eegfilt" (Delorme et al. 2004). A Hilbert transform was applied in order to estimate the envelope of fluctuations, followed by a smoothing of $256 \mathrm{~ms}$. In a second representation this transform was normalized by dividing the map obtained at a lower frequency band, as in (Bartolomei et al. 2008).

In order to compute connectivity graphs, we used the nonlinear correlation $h^{2}$ (van der Heyden et al. 1999; Wendling et al. 2000). Window size was set to 2 seconds, the maximum delay to $100 \mathrm{~ms}$, with a heuristic threshold set to 0.35 on the original signals and 0.15 on the despiked signals.

The robustness of the procedure was validated on a series of 100 realizations, in the two channels with a mixture of oscillations and spikes (channels 3 and 4). For each realization, we computed the correlation coefficient between the simulated data filtered in the band [35-95] $\mathrm{Hz}$ on the one hand and the simulated oscillations in the preictal phase on the other hand. This was done both on the original data and on the despiked data.

\section{Results}

\subsection{Simulated data}

We first tested the impact of the despiking procedure on the filtered data in the temporal domain, on one channel of simulated data (channel 4, presenting a mixture of spikes and oscillations at $85 \mathrm{~Hz}$ ). Results are presented in Figure 2. On the original signals (Fig 2a), the (sharp) spikes produced filter ringing (i.e. spurious oscillations) that arise from the impulse response of the filter (Fig. 2b), of shorter duration but higher amplitude than the actual oscillation. Such oscillations are often referred to as 'Gibbs effect' - although originally the Gibbs phenomenon was obtained from Fourier Series - and have been called 'false ripples' (Bénar et al. 2010) in the context of epilepsy. This implies that energy will be detected in the gamma band whenever spikes are present, whether accompanied by actual oscillations or not. In contrast, for the despiked signal (Fig2c), the impact of the high-frequency part of the spike has been drastically reduced (Fig 2 d). This implies that the filtering procedure will be much more specific for detecting actual oscillations, reducing the false detections produced 
by filter ringing. In supplementary Figure 2, we illustrate this effect for simulated oscillations with higher frequency (in the fast ripple band), in both a channel with a mixture of spikes and oscillation and a channel with no true oscillation.

We computed the ratio between the amplitude of the despiked signal versus that of the original signal, at the peak of the first (i.e., sharper) wave. The median ratio across channel, events and realizations was $4.6 \%$ (quartiles $1.7 \%-7.5 \%$ ), which means that most of the time of reduction of the spike of more than $90 \%$ was obtained. We did the same computation for the envelope of signals filtered in the $35-95 \mathrm{~Hz}$ band. In channel 1 , the median ratio was $48.9 \%$ (quartiles $47.8 \%$ - 50\%). In channel 2, with sharper spikes, the median ratio was 13.7 (quartiles $12.6 \%-15.3 \%$ ).

As the distinction between spikes and oscillations is often better done in the time-frequency plane (van der Heyden et al. 1999; Wendling et al. 2000; Bénar et al. 2010; Jmail et al. 2011), we studied the impact of despiking signals on the time-frequency representation of signals. An example is shown in Figure 3, corresponding also to channel 4. On the original signals (Figure 3a), spikes result in a wide-band pattern, and one can easily picture than depending on the time lag between spike and oscillations, the spike part could cover the 'blob' corresponding to the oscillation (Jmail et al. 2011). On the despiked signals (Figure 3b), the oscillatory part is more visible on the time-frequency representation.

A useful representation in clinical practice is based on a spatio-temporal map, where signals are filtered in a frequency band of interest (David et al 2011), with possible division by energy in low frequencies (Bartolomei et al 2008). This help mapping channels where the seizure starts, where typically there is a sudden increase in high frequency content and lowering of low frequencies (Bartolomei et al 2008). In Figure 4, we illustrate such representation on the simulated data, with or without despiking. On the original signal, when filtered in the frequency band that contains oscillations $(35-95 \mathrm{~Hz})$, a high energy level is visible on all channels presenting spikes, whether presenting actual oscillations or not (Fig. 4a). After despiking, there is better discrimination of channels with actual oscillations (Fig. 4b) - only little residuals from filtered spikes remain. When normalizing by low frequencies, all channels present low values, even when there are actual oscillations. Indeed, spikes present energy at all frequencies, and the division by energy in low frequencies thus 
prevents detecting high frequency activity (Fig. 4c). The best representations (in terms of specificity) are obtained for maps after despiking (Fig. $4 \mathrm{~b}$ and $4 \mathrm{~d}$ ).

Finally, we were interested in quantifying the gain in accuracy of reconstructing the oscillatory part of the signal obtained by the despiking procedure. We computed the correlation of both original signal and the despiked signal with the actual simulated oscillatory component, across 100 realizations and for the channels with oscillations (channels 3 and 4). The median correlation coefficient was 0.70 for the original signal (quartiles $0.57-0.82$ ) and 0.93 for the despiked signal (quartiles 0.92-0.94), showing an improvement which is both highly significant, and robust to different realizations of noise (Fig. 5).

\subsection{Real data}

Real data consisted of a section of intracerebral EEG data, in the period immediately preceding the epileptic seizure (i.e., the "preictal" period). In Figure 6, we show the spatiotemporal distribution of automatic detections. A large number of spikes were detected (range 0-82, median 34), mostly in the preictal phase and in most channels.

Figure 7 shows a time-frequency analysis in one channel presenting both spikes and oscillations, on a subset of the time window. One can observe that in the despiked signals there is almost no spike left; only oscillations with some slow waves (which do not impact time-frequency representation in the high frequency range) remain. In the time frequency representation of the despiked signal (Fig 7d), clear bursts of high frequency activity are now visible (indicated by white arrows), which were masked in the original representation (Fig 7d) due to the high frequency part of the spikes. To be noted, some real spikes can present larger slow waves than what we decided to include in our simulations (Figure 1 and 2). However, this has no impact in the frequency bands of interest (above $15 \mathrm{~Hz}$ ).

Finally, we computed the spatio-temporal maps on all channels, filtered in the $65-85 \mathrm{~Hz}$ band after normalization by the $8-30 \mathrm{~Hz}$ band (Figure 8). Interestingly, a buildup of gamma oscillations can be observed during the preictal period only in the despiked signals (Fig. 8b), whereas in the original signals this activity is masked by the high energy of the low frequency part of the spikes. Interestingly, several channels present energy in high frequency band 
during the seizure onset, but no preictal oscillations, which suggest that seizure onset and interictal oscillations may be different biomarkers of the epileptogenic zone.

\section{Discussion}

Much attention has been dedicated so far towards characterizing the initial part of the seizure, the so-called 'fast discharge' in SEEG. this activity is visible on the traces as a flattening with high frequency content (Wendling et al. 2000; Worrell et al. 2008). Bartolomei and colleagues have used a normalized estimate of energy, dividing the energy in the high frequencies $(12-97 \mathrm{~Hz})$ by that of low frequencies $(3.5-12 \mathrm{~Hz})$, which permits to emphasize the fast discharge (Bartolomei et al. 2008). David and colleagues (David et al. 2011) proposed to filter the traces in the gamma band only $(60-100 \mathrm{~Hz})$, based on the work of Worrell and colleagues (Worrell et al. 2008), and to map the energy content in 3D. Gnatkovsky and colleagues (Gnatkovsky et al. 2011) have presented a similar approach, using a higher frequency band $(110-125 \mathrm{~Hz})$.

When applied to the interictal state, where gamma band activity has been shown to be clinically relevant (Palmini et al. 2004), these two approaches (simple filtering or ratio of energies) present drawbacks due to the presence of spikes. Indeed, in the filtered preictal signal (when using the (David et al. 2011) approach), the sharp part of the spike appears as an energy increase in the gamma band, whether an actual oscillation is present or not (Bénar et al. 2010). This tends to overestimate the extent of regions presenting high frequency oscillatory activity. On the contrary, when normalizing by the low frequency content (as in (Bartolomei et al. 2008)), the low frequency part of the spike will tend to mask the oscillations, and underestimate the extent of oscillatory activity.

A solution for improving the estimation of high frequency oscillatory activity even in the presence of spikes is to separate the spiky and oscillatory parts of the signal (Jmail et al. 2011; Chaibi et al. 2013). Models that are too constrained may not model adequately the spike part (Jmail et al 2011). Here, we propose to fit in a nonlinear manner the spike patterns and to remove this model from the signals (Jmail et al 2015). We used a model consisting of a single wave, which has the advantage of being flexible enough to be applied to sharp artifacts or spikes that can consist of one, two or three waves. However, this may 
not be optimal as there could interference between consecutive waves modeled separately. More complex models could be designed, using Bayesian inference (which could incorporate constraints on the covariance between parameters), or adaptive methods based on dictionary learning (Hitziger et al. 2013). Another important factor, apart from correct modeling of the spike wave, is the detection of spikes. Many methods exist for automatic detection of spikes (review in (Harner 2009)), and it would be interesting to compare them in the context of spike removal.

We show in simulated data that this approach permits to retrieve the correct spatiotemporal dynamics of the gamma band activity. In the real SEEG data, this allowed revealing a build-up of gamma activity occurring just before the seizure. Removing the spikes also allows having a better time-frequency representation of the oscillatory activity, without being masked by the spike part (Amiri et al. 2015 ; Roehri et al.2016). Finally, it helps to better define the involved networks, which could be used in the level of graph definition and quantification by graph measures (Ponten et al. 2007).

The removal of the transient parts of the signal could be useful for other frequency bands, in order to better characterize the spatio-temporal dynamics of brain networks (Liu et al. 2015). Brain oscillatory networks in 'classical' frequency bands (from $1 \mathrm{~Hz}$ up to $120 \mathrm{~Hz}$ ) could also be contaminated by the band-pass filtering of transients, requiring an adequate pipeline for separation (Selesnick and Bayram 2009). High frequency oscillations (HFO, between $120 \mathrm{~Hz}$ and $500 \mathrm{~Hz}$ ) in the ripple and fast ripples bands (Urrestarazu et al. 2006; Chaibi et al. 2014) as well as physiological oscillations (Bragin et al. 2010; Waterstraat et al. 2016) could also benefit from such separation technique (see Supp Fig 2 for a preliminary test in the HFO frequency band).

Despiking could potentially be useful for other fields of neuroscience, where mixtures of transients and oscillations are present in the time series. For example, local field potentials present a mixture of oscillatory activity and sharp action potentials, and despiking could improve the investigation of correlations between LFP frequency bands and neuronal spiking (Mazzoni et al. 2010). Other modalities could also benefit from despiking, in particular EEG and MEG, where this could lead to a better characterization of the brain networks involved 
in oscillations (Lina et al. 2014), in the preictal state (Gavaret et al. 2004), but also in the interictal phase (Malinowska et al. 2013; Jmail et al.2016).

\section{Conclusion}

The correct mapping of the actual oscillatory activity, uncontaminated by spikes, seems a promising venue in delineating the hyperexcitable tissues on the preictal signals. Such despiking should allow mapping separately the dynamics of the spikes and those of gamma oscillations, and thus to compare their clinical values as biomarkers. 
Figures (screen captures, source files are of better quality)

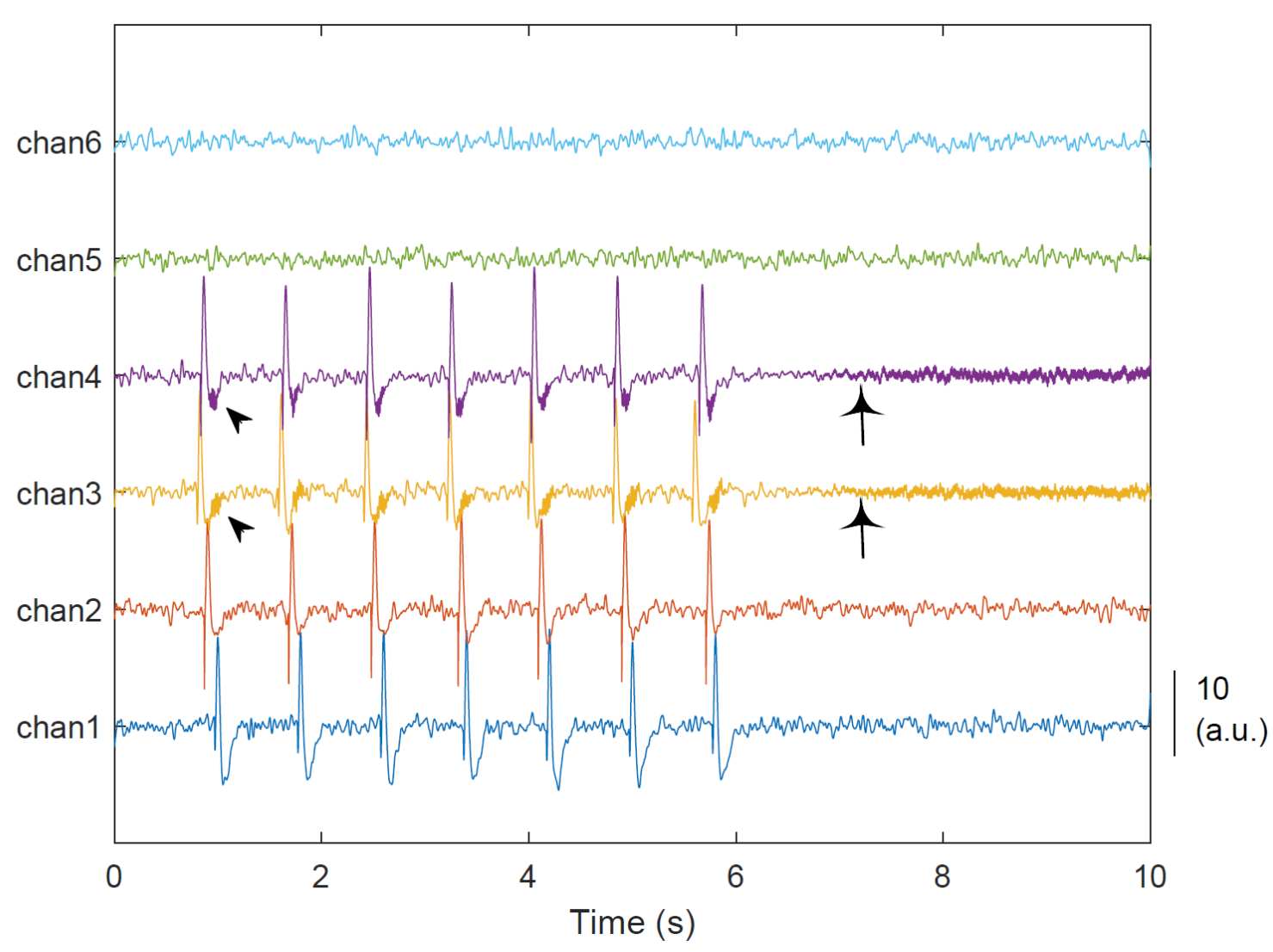

Figure 1: One realization of simulated data, reproducing a preictal spiking pattern in channels 1-4 (between $t=0 \mathrm{~s}$ and $t=6 \mathrm{~s}$ ) followed by an ictal-like discharge in channels 3 and 4 (the simulated seizure start is at $t=7 \mathrm{~s}$, indicated by two vertical arrows). In channels 3 and 4 , oscillations were added to each spike (oscillations in the first spikes are indicated by tilted arrow heads). 
(a)

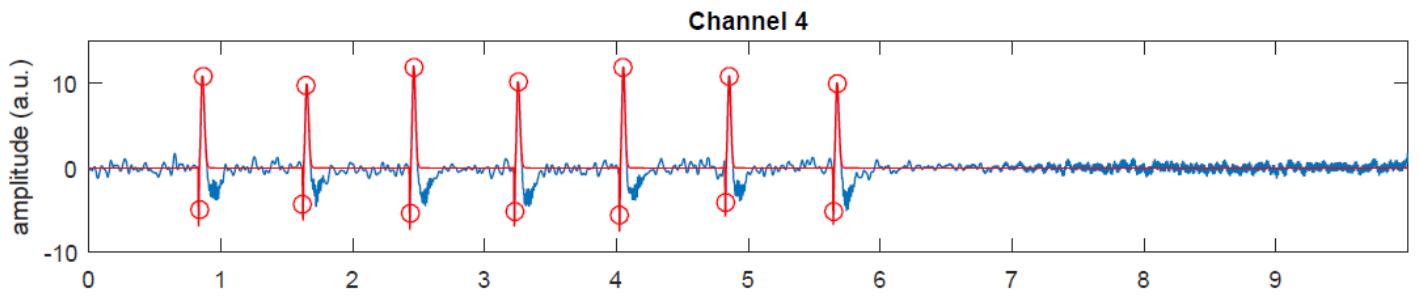

(b)

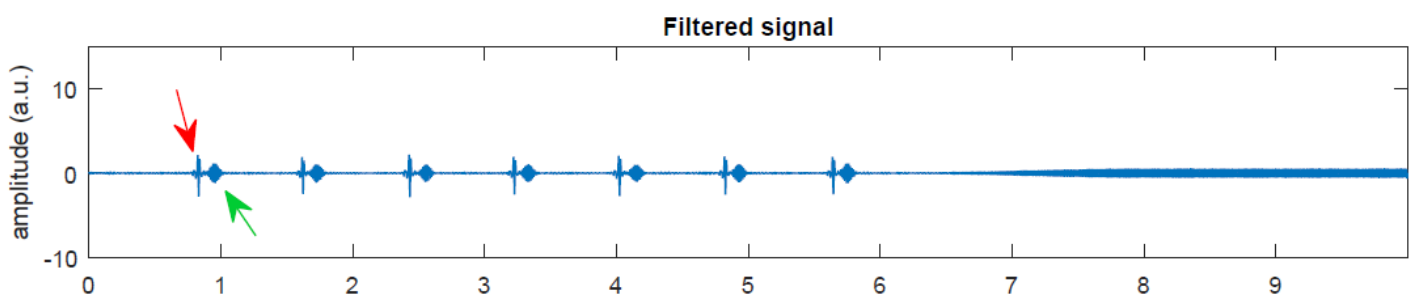

(c)

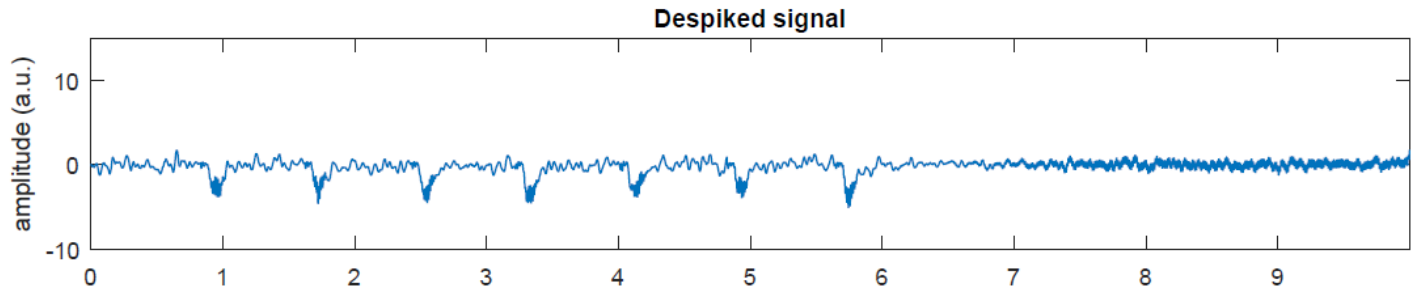

(d)

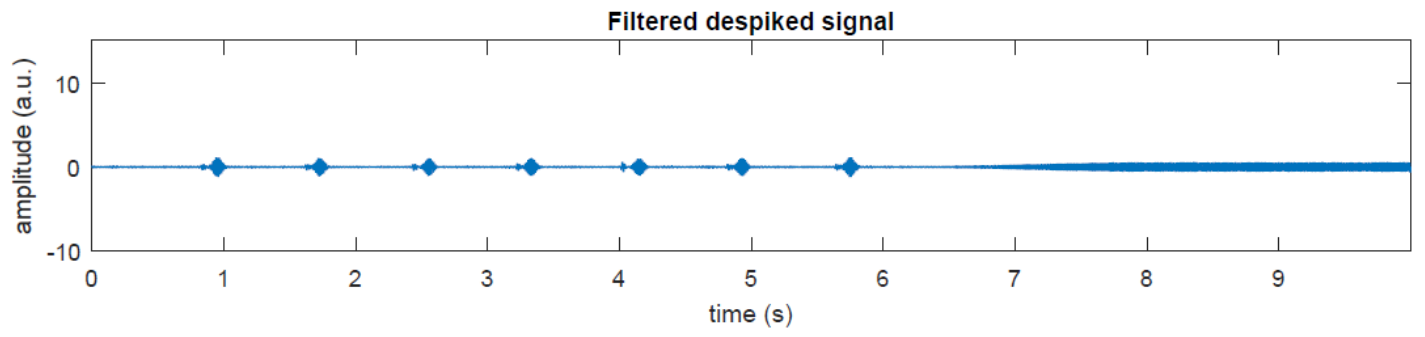

Figure 2: Illustration of the despiking procedure. a) Simulated data (in blue, corresponding to channel 4 of Fig. 1) along with modeled spikes (in red). Spikes detections are shown as red circles. The model is a set of modified (asymmetric) Gaussian functions. b) Same signal filtered in the $35-95 \mathrm{~Hz}$ (gamma) band. Strong filter-related ringing at the time of spikes are visible (red arrow), which correspond to the impulse response of the filter. The ringing waveforms have higher amplitude than the actual oscillations present in the signal (green arrow). c) Despiked signal, obtained by subtracting the modeled spikes from the original signal. The slow components remain, along with actual oscillations d). Same signal filtered in the $35-95 \mathrm{~Hz}$ band. The true oscillations (within spikes, as well as the ictal discharge) are now clearly extracted; only a small residual from filtered spikes remain. 

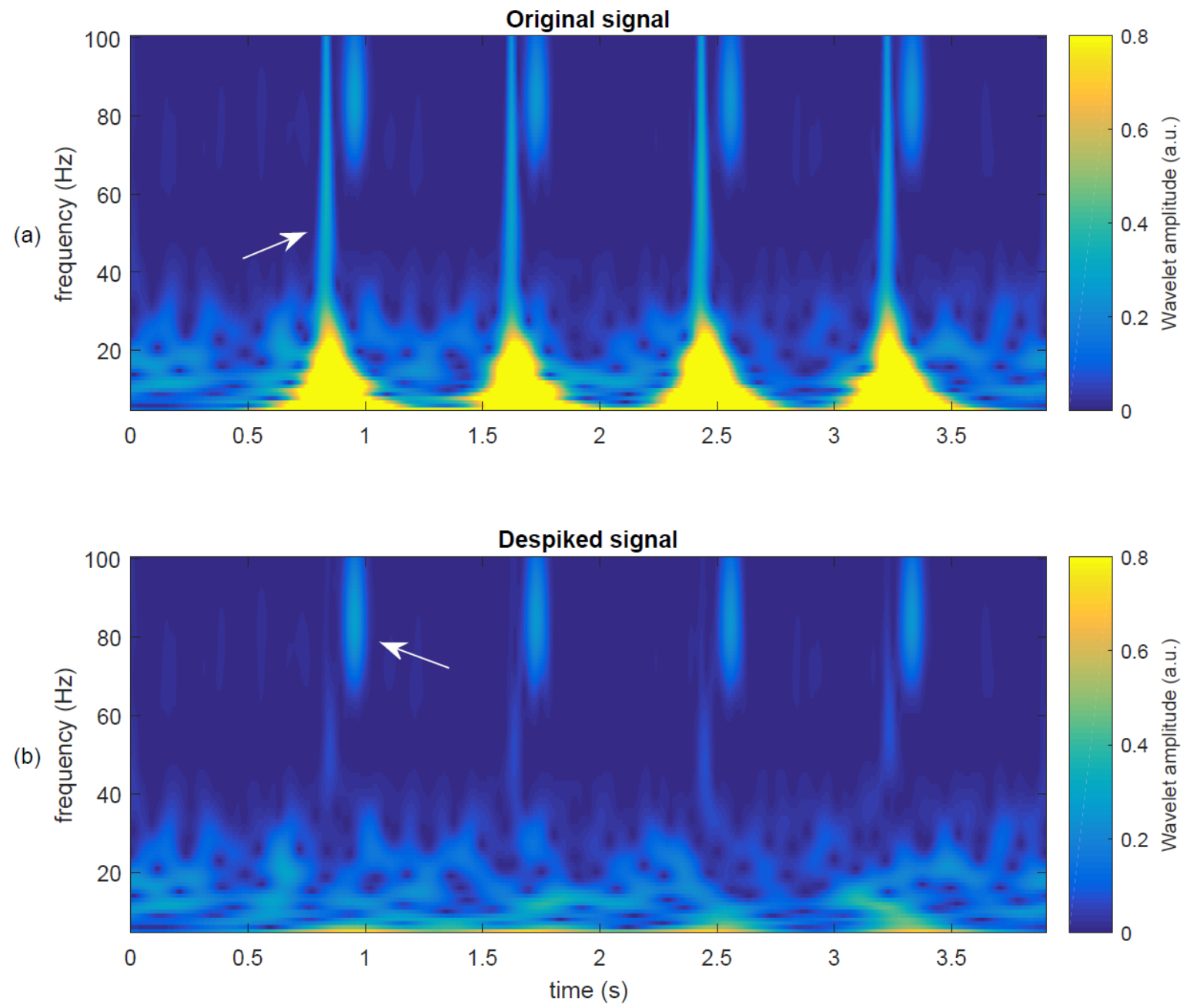

Figure 3: Time frequency analysis of simulated data (same data than Fig. 2). a) Original signal. In the time frequency plane, spikes present energy at all frequencies, producing an horizontal line (arrow). b) Despiked signal. The actual oscillations, corresponding to "blobs" in the time frequency plane (white arrow), now stand out better within their own frequency band. 
(a)

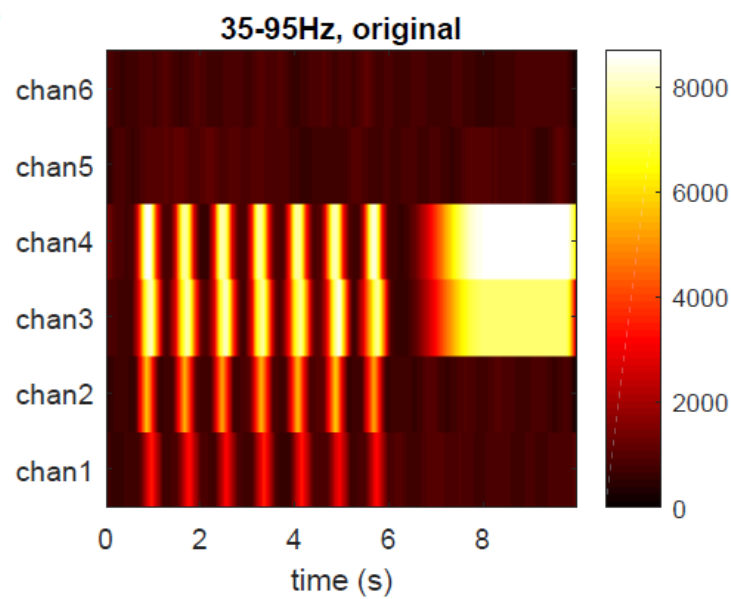

(c)

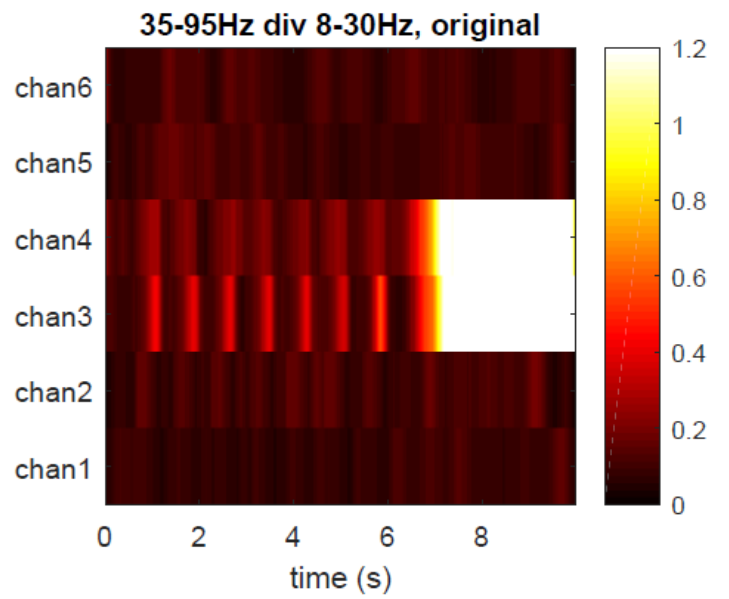

(b)

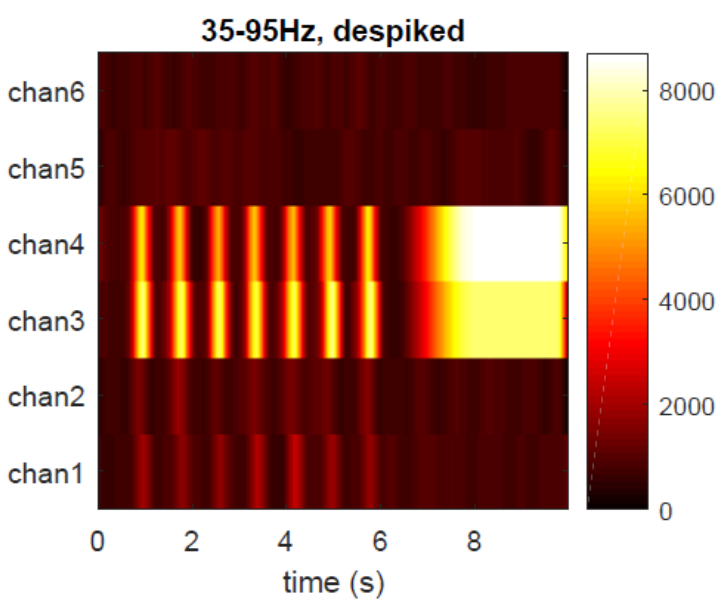

(d)

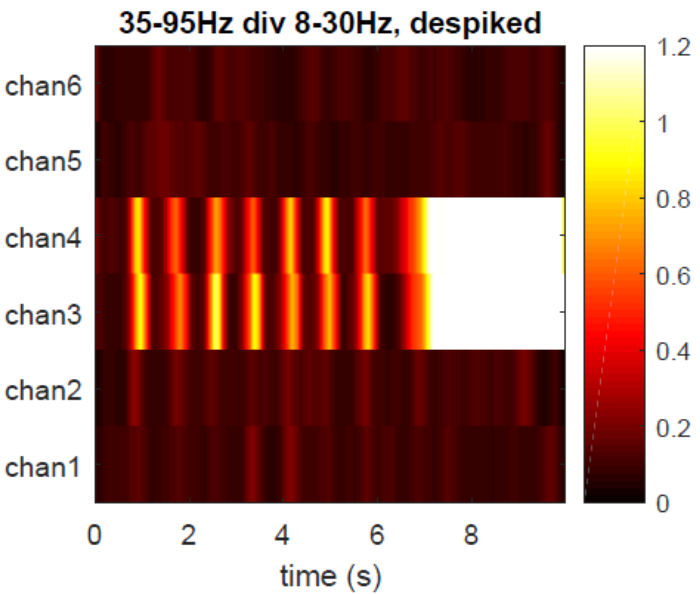

Figure 4: Spatio-temporal maps (channels $x$ time) of energy for the simulated data. a) Original signals, filtered in the $35-95 \mathrm{~Hz}$ band. In the preictal period $(0-6 \mathrm{~s})$, there is high energy visible in channels 1-4, corresponding to both channels with a mixture of spikes and true oscillations (channels 3 and 4) and to channels with only spikes (channels 1-2). B) Despiked signals. Mostly channels with true oscillations (channels 3-4) remain visible. c) Original signal with normalization by low frequencies $(8-30 \mathrm{~Hz})$. As spikes have energy across all frequencies, all channels have low energy values. d) Despiked signal with normalization. 


\section{Correlation with simulated oscillatory part}

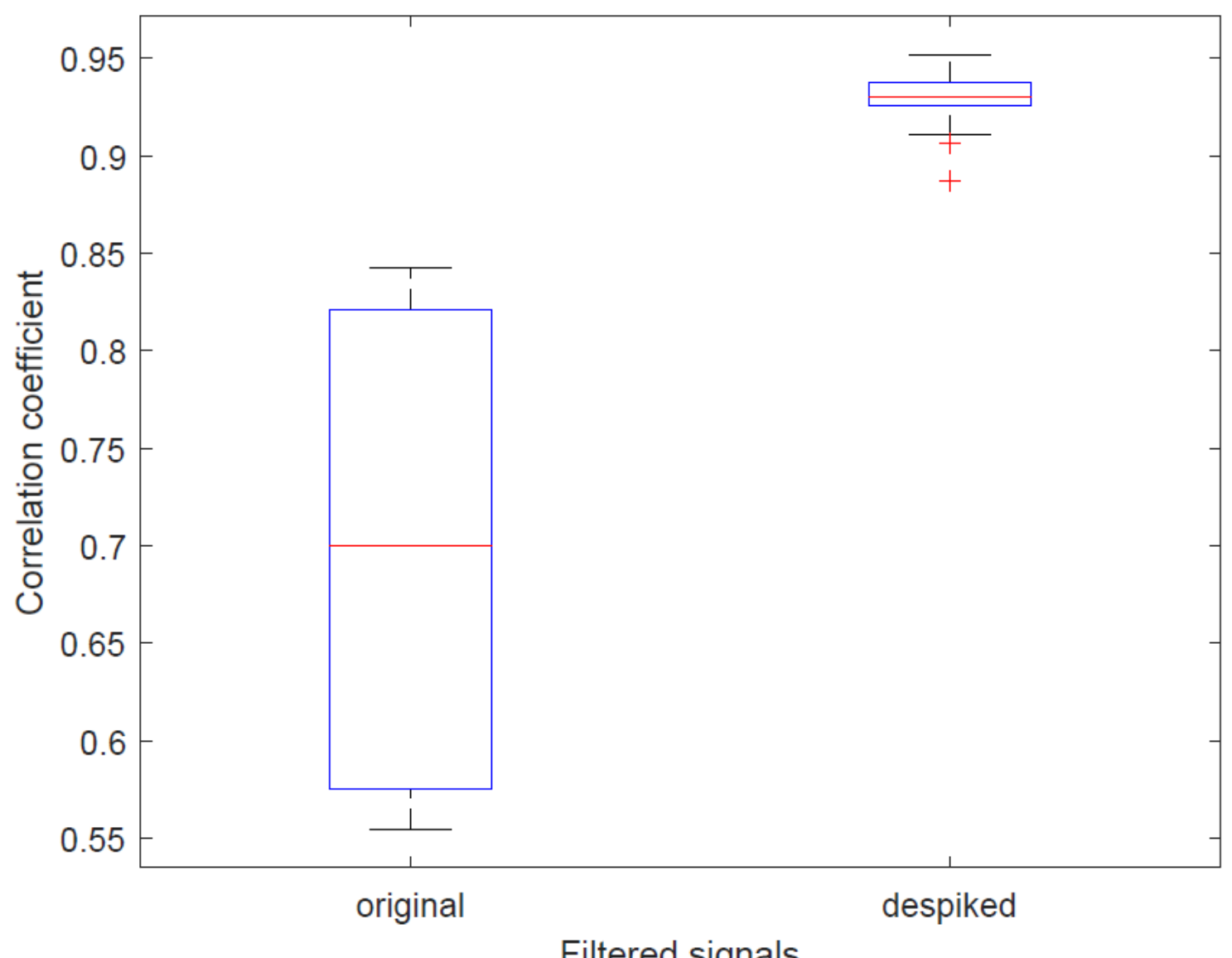

Filtered signals

Figure 5: Boxplots of the correlation coefficients between filtered data and the (pure) simulated oscillations, across 100 realizations of the simulated signals. On each box, the central mark is the median, the edges of the box are the 25th and 75th percentiles, the whiskers extend to the most extreme data points not considered outliers, and outliers are plotted individually.On the original signals (left boxplot), the spurious oscillations arising from filtered spikes (filter ringing) result in low correlation values. On the despiked signals (right boxplot), correlation values are high, showing that the actual oscillatory part of signals were recovered. 


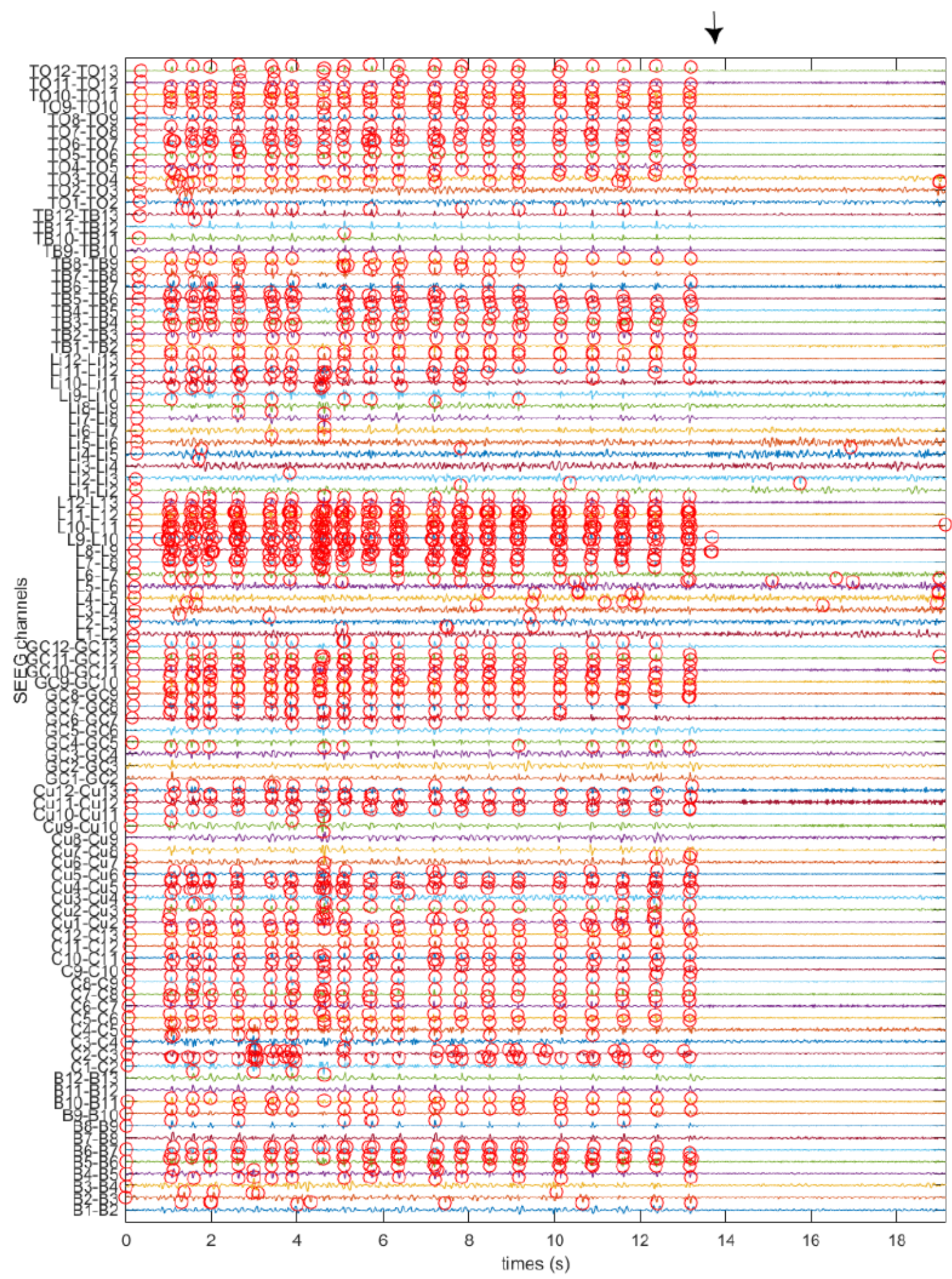

Figure 6: Results of automatic spike detection, performed in the preictal period and the seizure start, on the real SEEG signals. Seizure onset is marked by a vertical arrow. There is continuous spiking in the preictal period (from 0 to $14 \mathrm{~s}$ ), in most channels. 

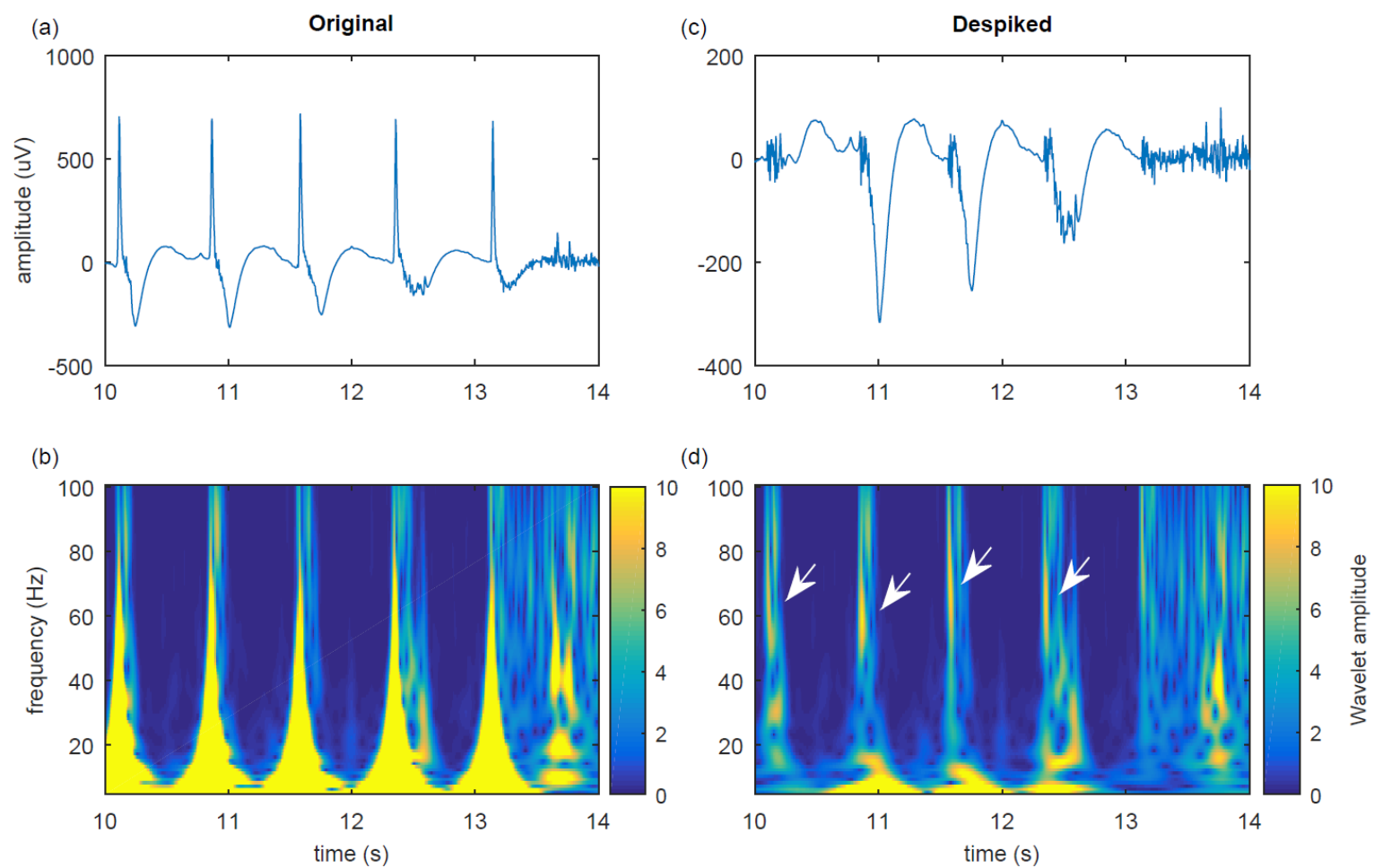

Figure 7: Time-frequency analysis in one channel (L9-L10), on a subset of the time window (from 10 to 14s). a) Original signal. Five high-amplitude spikes can be seen. Preictal oscillations are visible in the slow part spikes, especially for 4th one. b) Time frequency plane. Spikes produce high amplitude pyramidal-like shapes which hampers the visibility of oscillations. c) Despiked signal. Oscillations are more clearly visible already on the timedomain signal. d) Time frequency representation of the despiked signals. Oscillations can now be seen as 'blobs' (arrows). 

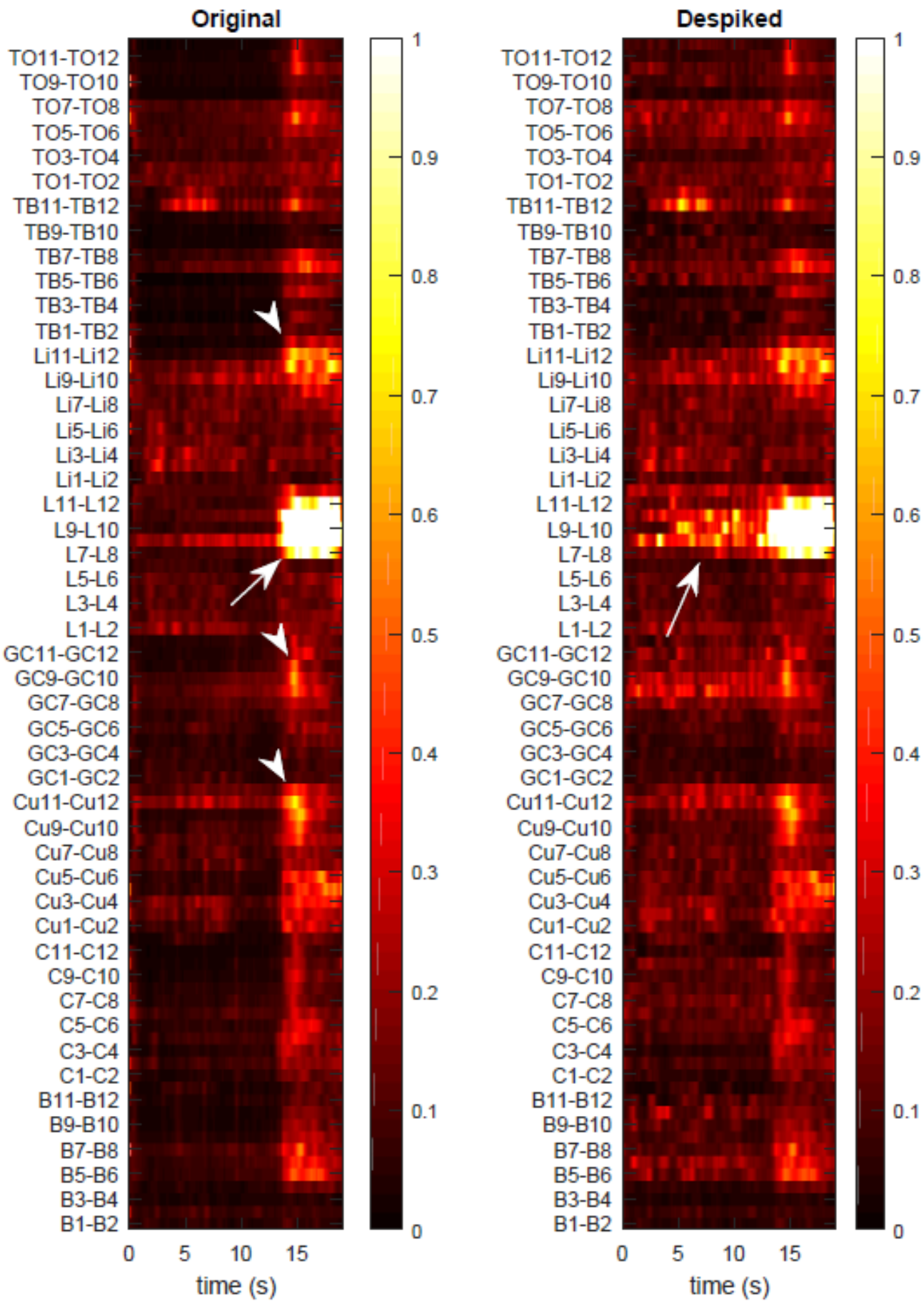

Figure 8: Spatio-temporal maps of real signals (channels $x$ time), filtered in the $65-85 \mathrm{~Hz}$ band with normalization by the $8-30 \mathrm{~Hz}$ band and smoothing by a moving average of $500 \mathrm{~ms}$. a) Original signals There is no clear pattern in the preictal period (between 0 and 14s). Seizure start can be seen in several regions (arrows), with maximum energy ratio in channels L7-L8 to L11-L12 (arrow with tail). b) Despiked signals. Thanks to the decrease in the energy resulting from filtering spikes, a clear 'build-up' of oscillatory activity that leads to the seizure are now seen in channels L8-L9 to L10-L11 (arrow with tail). 
Acknowledgements: The authors thank the anonymous reviewers for their help on improving the manuscript. CGB thanks Patrick Chauvel for useful discussions on preictal buildup of gamma oscillations. Part of this work was funded by a joint grant from Agence Nationale de la Recherche (ANR) and Direction Génerale de I'Offre de Santé (DGOS), "VIBRATIONS" ANR 13 PRTS 0011 01. This work has been carried out within the FHU EPINEXT with the support of the A*MIDEX project (ANR-11-IDEX-0001-02) funded by the "Investissements d'Avenir" French Governement program managed by the French National Research Agency (ANR).

\section{References}

Amiri M, Lina J-M, Pizzo F, Gotman J. High Frequency Oscillations and spikes: Separating real HFOs from false oscillations. Clin Neurophysiol. 2015.

Ayoubian L, Lacoma H, Gotman J. Automatic seizure detection in SEEG using high frequency activities in wavelet domain. Med Eng Phys . Institute of Physics and Engineering in Medicine; 2013;35(3):319-28.

Bartolomei F, Chauvel P, Wendling F. Epileptogenicity of brain structures in human temporal lobe epilepsy: A quantified study from intracerebral EEG. Brain. 2008;131(7):1818-30.

Bénar CG, Papadopoulo T, Torrésani B, Clerc M. Consensus Matching Pursuit for multi-trial EEG signals J Neurosci Methods. 2009 ;180(1):161-70.

Bénar CG, Chauvière L, Bartolomei F, Wendling F. Pitfalls of high-pass filtering for detecting epileptic oscillations: A technical note on "false" ripples. Clin Neurophysiol. 2010;121(3):301-10.

Bragin A, Engel J Jr , Staba R J. High-frequency oscillations in epileptic. brain Curr. Opin. Neurol.2010; $23,151-6$.

Chaibi S, Lajnef T, Sakka Z, Samet M, Kachouri A. A reliable approach to distinguish between transient with and without HFOs using TQWT and MCA. J Neurosci Methods Elsevier B.V.; 2014;232:36-46.

Chaibi S, Sakka Z, Lajnef T, Samet M, Kachouri A. Automated detection and classification of 
high frequency oscillations (HFOs) in human intracereberal EEG. Biomed Signal Process Control. Elsevier Ltd; 2013;8(6):927-34.

Crépon B, Navarro V, Hasboun D, Clemenceau S, Martinerie J, Baulac M, et al. Mapping interictal oscillations greater than $200 \mathrm{~Hz}$ recorded with intracranial macroelectrodes in human epilepsy. Brain. 2010;133:33-45.

David O, Blauwblomme T, Job AS, Chabards S, Hoffmann D, Minotti L, et al. Imaging the seizure onset zone with stereo-electroencephalography. Brain. 2011;134(10):2898-911.

Delorme A, Makeig S. EEGLAB: an open source toolbox for analysis of single-trial EEG dynamics including independent component analysis. J Neurosci Methods. 2004 $15 ; 134(1): 9-21$.

Gavaret M, Badier JM, Marquis P, Bartolomei F, Chauvel P. Electric source imaging in temporal lobe epilepsy. J Clin Neurophysiol. 2004;21:267-82.

Gnatkovsky V, Francione S, Cardinale F, Mai R, Tassi L, Lo Russo G, et al. Identification of reproducible ictal patterns based on quantified frequency analysis of intracranial EEG signals. Epilepsia. 2011;52(3):477-88.

Gotman J, Gloor P. Automatic recognition and quantification of interictal epileptic activity in the human scalp EEG. Electroencephalogr Clin Neurophysiol . 1976;41(5):513-29.

Harner R. Automatic EEG spike detection. Clin EEG Neurosci. 2009 ;40(4):262-70.

Hirai N, Uchida S, Maehara T, Okubo Y, Shimizu H. Enhanced gamma $(30-150 \mathrm{~Hz})$ frequency in the human medial temporal lobe. Neuroscience. 1999;90(4):1149-55.

Hitziger S, Clerc M, Gramfort A, Saillet S, Benar CGB, Papadopoulo. Jitter-Adaptive Dictionary Learning -Application to Multi-Trial Neuroelectric Signals.ICLR - 1st International Conference on Learning Representations .2013, United States.

Jacobs J, LeVan P, Chander R, Hall J, Dubeau F, Gotman J. Interictal high-frequency oscillations $(80-500 \mathrm{~Hz})$ are an indicator of seizure onset areas independent of spikes in the human epileptic brain. Epilepsia. 2008;49(11):1893-907.

Jmail N, Gavaret M, Wendling F, Kachouri A, Hamadi G, Badier JM, et al. A comparison of methods for separation of transient and oscillatory signals in EEG. J Neurosci Methods. 2011;199(2):273-89. 
Jmail N, Gavaret M, Bartolomei F, Bénar CG. Despikifying SEEG signals using a temporal basis set. The International Conference on Intelligent Systems Design and Applications 2015.

Jmail N, Gavaret M, Bartolomeil F, Chauvel P, Badier JM, Bénar CG . Comparison of Brain Networks During Interictal Oscillations and Spikes on Magnetoencephalography and Intracerebral EEG. Brain Topogr. 2016 ;29(5):752-65.

Lina JM, Chowdhury R, Lemay E, Kobayashi E, Grova C. Wavelet-based localization of oscillatory sources from magnetoencephalography data. IEEE Trans Biomed Eng. IEEE Computer Society; 2014;61(8):2350-64.

Liu L K K, Bartsch R P, Lin A, Mantegna RN, Ivanov PC .Plasticity of brain wave network interactions and evolution across physiologic states . Frontiers in Neural Circuits, 2015; 9:62.

Malinowska U, Badier JM, Gavaret M, Bartolomei F, Chauvel P, Bénar CG. Interictal networks in Magnetoencephalography. Hum Brain Mapp. 2013;2805:2789-805.

Mazzoni A, Whittingstall K, Brunel N, Logothetis NK, Panzeri S. Understanding the relationships between spike rate and delta/gamma frequency bands of LFPs and EEGs using a local cortical network model. Neuroimage. 2010;52(3):956-72.

Palmini A, Najm I, Avanzini G, Babb T, Guerrini R, Foldvary-Schaefer N, et al. Terminology and classification of the cortical dysplasias. Neurology. 2004;62(6 Suppl 3):S2-8.

Ponten SC, Bartolomei F, Stam CJ. Small-world networks and epilepsy: graph theoretical analysis of intracerebrally recorded mesial temporal lobe seizures. Clin Neurophysiol . 2007;118(4):918-27. 8.

Roehri N, Lina JM, Mosher JC, Bartolomei F, Benar CG. Time-frequency strategies for increasing high frequency oscillation detectability in intracerebral EEG. IEEE Transactions on Biomedical Engineering Year: 2016

Schwartz TH, Hong SB, Bagshaw AP, Chauvel P, Bénar CG. Preictal changes in cerebral haemodynamics: Review of findings and insights from intracerebral EEG. Epilepsy Res. Elsevier B.V.; 2011;97(3):252-66.

Selesnick IW, Bayram I. Oscillatory plus transient signal decomposition using overcomplete rational-dilation wavelet transforms. Proc SPIE. 2009;7446:7446. 
Talairach J, Bancaud J. tereotaxic approach to epilepsy. Methodology of anatomo-functional stereotaxic investigations. Prog Neurol Surg. 1973;5:297-354.

Urrestarazu E, Chander R, Dubeau F, Gotman J. Interictal high-frequency oscillations (100$500 \mathrm{~Hz}$ ) in the intracerebral EEG of epileptic patients. Brain. 2007;130(Pt 9):2354-66.

Urrestarazu E, Jirsch JD, LeVan P, Hall J, Gotman J. High-frequency intracerebral EEG activity (100-500 Hz) following interictal spikes. Epilepsia. 2006;47(9):1465-76.

van der Heyden MJ, Velis DN, Hoekstra BP, Pijn JP, van Emde Boas W, van Veelen CW, et al. Non-linear analysis of intracranial human EEG in temporal lobe epilepsy. Clin Neurophysiol. 1999;110(10):1726-40.

Waterstraat G, Scheuermann M, Curio G. Non-invasive single-trial detection of variable population spike responses in human somatosensory evoked potentials. Clin Neurophysiol; 2016; 127(3):1872-8

Wendling F, Bartolomei F, Bellanger JJ, Bourien J, Chauvel P. Epileptic fast intracerebral EEG activity: Evidence for spatial decorrelation at seizure onset. Brain. 2003;126(6):144959.

Wendling F, Bellanger JJ, Bartolomei F, Chauvel P. Relevance of nonlinear lumped-parameter models in the analysis of depth-EEG epileptic signals. Biol Cybern. 2000;83(4):367-78.

Wendling F, Hernandez A, Bellanger J-J, Chauvel P, Bartolomei F. Interictal to ictal transition in human temporal lobe epilepsy: insights from a computational model of intracerebral EEG. J Clin Neurophysiol. 2005;22(5):343-56.

Worrell GA, Gardner AB, Stead SM, Hu S, Goerss S, Cascino GJ, et al. High-frequency oscillations in human temporal lobe: Simultaneous microwire and clinical macroelectrode recordings. Brain. 2008;131(4):928-37. 